\title{
EL PAPEL DEL RECONOCIMIENTO EN LA INTEGRACIÓN SOCIAL DE LAS PERSONAS DISCAPACITADAS: ANÁLISIS DE MODELOS DE INSERCIÓN LABORAL PARA PERSONAS CIEGAS EN EL ÁREA METROPOLITANA DE MONTERREY
}

\author{
THE ROLE OF RECOGNITION IN THE SOCIAL INTEGRATION OF DISABLED PERSONS: \\ ANALYSIS OF MODELS OF EMPLOYMENT FOR BLIND PEOPLE IN THE METROPOLITAN \\ AREA OF MONTERREY
}

\section{Brenda Araceli Bustos $G^{1}$}

\begin{abstract}
Resumen
Partiendo de la tesis de que el reconocimiento es una precondición para una vida en sociedad (Honneth, 2010:32) debido a que: a) hace posible la construcción de la identidad; b) incide en la relación con uno mismo; c) es el fundamento de la empatía y solidaridad; d) incide en la valoración social de una actividad; e) y afecta incluso la distribución de bienes materiales (Honneth, 2010: 43). Consideramos que la forma como son reconocidos los diversos grupos sociales influye en la valoración de sus actividades, cualidades y aportaciones sociales, organiza y determina su acceso a la distribución de los bienes materiales socialmente disponibles. Desde esta perspectiva es que analizamos los datos empíricos obtenidos a través de la elaboración de entrevistas a profundidad a personas ciegas del AMM; y qué organismos a partir de cómo los modelos de inserción laboral desarrollados por las distintas Asociaciones de las que los entrevistados forman parte materializan, en sus prácticas de inserción, un determinado imaginario social sobre las personas ciegas. Asimismo consideramos que ese imaginario social incide en la autopercepción de las mismas personas. Finalmente concluimos que las personas con discapacidad enfrentan una especie de negación de reconocimiento con relación a sus cuerpos. Ello inhibe y reprime el desarrollo de sus capacidades y habilidades para conocer y aprehender el mundo.
\end{abstract}

\section{Palabras clave:}

Reconocimiento; modelos de inserción laboral; personas ciegas; políticas identitarias

\begin{abstract}
Starting from the thesis that recognition is a precondition for a life in society (Honneth, 2010:32) because: a) makes possible the construction of identity; b) affects the relationship with oneself; c) is the foundation of empathy and solidarity; d) affects the social value of an activity; e) and affects even distribution of material goods (Honneth, 2010: 43). Consider how they are recognized various social groups affect the value of their activities, qualities and social contributions, organizes and determines their access to distribution of material goods socially available. From this perspective, we analyze the empirical data obtained through the development of in-depth interviews to blind the AMM and how organisms from employment models developed by different organizations which respondents are part materialize, insertion practices in a particular social imaginary of the blind. We also believe that social
\end{abstract}

1 Dra. En Filosofía con Orientación en Trabajo Social y Políticas Comparadas de Bienestar Social. Docente - investigadora de tiempo completo Facultad de Filosofía y Letras, UANL, México. Correo electrónico: brendaaraceli2001@hotmail.ocm 
imagery affects self-perception of the same people. Finally we conclude that persons with disabilities we face a kind of denial of recognition in relation to their bodies. This inhibits and suppresses the development of their skills and abilities to appreciate and understand the world.

\section{Key words:}

Recognition; employment models; blind people; identity politics

\section{INTRODUCCIÓN}

\section{Reconocimiento e identidad}

Honneth es uno de los primeros sociólogos que retoman el concepto de reconocimiento para analizar las problemáticas de integración e inclusión no sólo en cuestiones materiales (empleo, educación, etcétera) sino para utilizarlo en el ámbito de la interacción social y como punto inicial de ésta.

La propuesta de Honneth se caracteriza por cuestionar la tesis clásica de que la intersubjetividad surge a partir de un individuo que busca conocer a otro. Según Honneth (2007: 49), la intersubjetividad está precedida por una actitud de reconocimiento hacia el otro. El autor agrega que: a) éste es el primer momento de la interacción; b) y que se tratará de un momento que no admite diferenciación entre elementos emocionales, cognitivos, etc. (Honneth, 2007: 54). Honneth (2010) considera que el reconocimiento es una manifestación de la aceptación, inclusión e integración social de la persona, por lo que se convierte en una necesidad fundamental en la existencia social de cualquier individuo (Basaure 2011: 77).

De esta manera la tesis de Honneth gira en torno al planteamiento de que el reconocimiento de nuestras particularidades, de nuestra personalidad, es sustancial para la integración social, es decir, para identificarnos y considerarnos como miembros de la sociedad. Además, agrega, existe una conexión obligatoria entre el reconocimiento intersubjetivo y la autoconciencia. Por lo que es imprescindible para la salud psíquica de los individuos y para el logro de la autonomía individual (Basaure, 2011: 77).
Finalmente podríamos señalar que, para Honneth, el imaginario social en torno a los grupos sociales, por ejemplo personas discapacitadas, se materializará en la valoración de las actividades, cualidades y aportaciones sociales que éstos pueden realizar. De esta manera, dichos imaginarios determinan las normas que organizan la distribución de bienes materiales (Honneth, 2010: 41).

En suma, el reconocimiento es una precondición para una vida en sociedad (Honneth, 2010:32) debido a que: a) hace posible la construcción de la identidad; b) incide en la relación con uno mismo; c) es el fundamento de la empatía y solidaridad; d) incide en la valoración social de una actividad; e) y afecta incluso la distribución de bienes materiales (Honneth, 2010: 43). Podemos señalar que, para Honneth (2010: 43), la forma como son reconocidos los diversos grupos sociales influye en la valoración de sus actividades, cualidades y aportaciones sociales, organiza y determina su acceso a la distribución de los bienes materiales socialmente disponibles.

La tesis de Honneth acerca del reconocimiento ha sido criticada por Ricoeur, quien considera que esta perspectiva de análisis da por supuesta una reciprocidad en la construcción del reconocimiento mutuo (Ricoeur, 2006). En contrapartida, Ricoeur asegura que el reconocimiento se construye desde una disimetría originaria entre el yo y el otro (Ricoeur, 2006). Dado que la vivencia del otro me resulta inaccesible, jamás podré experimentarla. El otro es aprehendido solamente de manera analogizante (Ricoeur 2006: 199), la cual es construida como una trasposición de mi propia experiencia, como primera creación de sentido, hacia el otro: 
"hace de la relación de mí con lo extraño una relación de modelo con la copia"(Ricoeur, 2006: 199).

Una de las preguntas planteadas por Ricoeur es ¿cómo comparar incomparables con el fin de igualarlos? Ante esta situación, se encuentra latente la amenaza de no terminar con el desconocimiento, de llegar a encontrarse en una situación de negación de reconocimiento (Ricoeur, 2006: 207). El relacionarse con otros seres humanos, el vivir en comunidad, se convierte en una fuente que conlleva la amenaza latente de negación de reconocimiento por parte de los otros: tanto de los sujetos particulares como de las instituciones sociales y el propio Estado. En este sentido, como plantea Ricoeur, la problemática en torno al reconocimiento es de naturaleza política. Finalmente, Ricoeur señala que el ser reconocido implica:

"recibir la plena garantía de su identidad gracias al reconocimiento por parte de otro de su dominio de capacidades" (2006:312).

Esta perspectiva de análisis posibilita el abordaje de los denominados grupos vulnerables bajo otra óptica. Podríamos decir que la vulnerabilidad atribuida se construye desde capacidades que se han vuelto hegemónicas, por ejemplo, las físicas. En el caso de las personas ciegas, lo anterior significa que sus propias capacidades de conocer el mundo a través de medios no-visuales, de interactuar con y desenvolverse en el mismo, no son reconocidas.

\section{Metodología}

Para el desarrollo del presente estudio se eligió un enfoque cualitativo, debido a que nos interesaba conocer la relación entre imaginario social, formaciones identitarias y agencia social entre invidentes del Área Metropolitana de Monterrey (AMM). Los nombres con los que se identifica a los entrevistados no son reales, esto con el fin de proteger su anonimato .

\section{El instrumento de recopilación de datos}

A través de entrevistas en profundidad se intentó recopilar las experiencias de los invidentes en cuanto a la exclusión social y laboral. La hipótesis inicial contenida en la guía de entrevista consistió en proponer que, la discriminación se articula en gran medida a través de la deficiente infraestructura urbana (por ejemplo, ausencia de semáforos para invidentes en las cruces de avenidas y calles). Sin embargo, pronto se reconoció que para los discapacitados la exclusión laboral y educativa pesaba mucho más que los problemas de infraestructura por lo que las entrevistas se enfocaron en mayor grado en las dificultades más sentidas por los informantes.

En el mismo sentido, se invirtió el orden de las preguntas seleccionadas con otro punto clave: la exploración de las estrategias desarrolladas para superar la discriminación y la exclusión social. Se confirmó así la prioridad de los discapacitados, los informantes enfatizaban en mucho mayor grado las estrategias ligadas al estudio y el empleo, por lo cual la guía de entrevista integró un mayor número de preguntas al respecto.

La guía de entrevista se aplicó a un total de diez personas. Seis entrevistas se realizaron de forma individual y cuatro en grupo. En una de ellas participaron cuatro personas; la segunda entrevista grupal se realizó durante una clase para entrenar a bebes invidentes. En ella participaron la maestra y algunas madres de familia. Otras dos entrevistas grupales se efectuaron con dos personas respectivamente.

\section{Interpretación de los datos}

El primer paso para la interpretación fue la transcripción de las entrevistas realizadas. La transcripción respetó los modismos empleados por los informantes, es decir fue literal. Cuando la entrevista se encontraba en archivo Word, se procedió a nume- 
rar las páginas y líneas de texto (Martínez, 2005: 7) con el fin de identificar palabras clave.

El siguiente paso consistió en la identificación de unidades temáticas (Martínez, 2005: 8) mediante palabras clave y párrafos que abordaron o refirieran a un concepto central. A partir de estos conceptos se crearon categorías de análisis como, por ejemplo, 'estudio' 'estrategias', 'empleo', 'desplazamiento', trayectoria de la enfermedad (avance de la pérdida de la vista hasta llegar a la ceguera), desplazamiento, círculo social, compañero de trabajo, encuentros con transeúntes, etcétera. Cada categoría central fue dividida después en sub-categorías: por ejemplo, la categoría de 'estudio' se subdividió en 'tecnología didáctica', 'cursos', 'relación con compañeros'.

Para el análisis del discurso se retomó la obra de Renkema (1999), en particular sus observaciones acerca de la deixis. Dado que la deixis hace referencia al sujeto del discurso: su sentido de pertenencia a través de la deixis de la persona construida mediante los pronombres personales; su ubicación en el tiempo (a través de adverbios del tiempo y tiempo verbales) y su locación en el espacio 'real' y simbólico (a través de adverbios locales).

El análisis de la deixis resulta central en un estudio que analiza formaciones identitarias y la relación entre sujetos y entorno social. Por ejemplo, el análisis del campo deíctico indica cómo los sujetos entrevistados percibieron y construyeron la exclusión social, cómo construyeron a otros grupos de invidentes, el tipo de relación que sostienen con ellos o el grado de integración social a la sociedad.

\section{Resultados}

\section{Algunas instituciones de atención a invidentes en Nuevo León}

La atención a invidentes en Nuevo León se guía en varios tipos de instituciones con características particulares y que se pueden dividir de la siguien- te manera: a) instituciones que ofrecen apoyo técnico a los invidentes; b) instituciones que ofrecen entre su variada gama de servicios sociales atención a la discapacidad en general; c) instituciones creadas especialmente para la atención de invidentes que intentan combinar, en diversos grados, la asistencia educativa, social y médica con la integración laboral; d) un modelo cooperativista de integración económica y social que ha surgido últimamente entre grupos de invidentes.

En las siguientes líneas presentaremos las características generales de los diversos enfoques de intervención tal como son percibidos por los invidentes que participan en ellos en calidad de 'beneficiarios' u como organizadores.

Entre las instituciones que ofrecen servicios de apoyo técnico se encuentran la Universidad Autónoma de Nuevo León y la Biblioteca Fray Servando Teresa de Mier. La UANL ofrece a los invidentes una sala tiflotécnica que cuenta con computadoras e impresoras para documentos en braille. Sin embargo, ante la falta de personal especializado y la falta de difusión, el alcance de estos servicios se ve disminuido. En la Biblioteca Fray Servando existe una sala de lectura para invidentes.

El Club de Leones es una de las instituciones más antiguas que ofrece atención general a personas con una discapacidad. En sus inicios se enfocó solamente a ciegos (http://www.lionsclubs. org), pero con el tiempo ha ampliado sus servicios, atendiendo hoy en día una gama mayor de discapacidades a través del Centro de Atención Múltiple². En las mañanas se atienden a los niños y por la tarde a los adultos. Dicho centro brinda servicios educativos y consultas. En ocasiones, el Club de Leones entrega donativos a otras asociaciones que atienden la discapacidad.

En el caso de los bebés y los niños del nivel pre-escolar se práctica la intervención temprana

2 Andrea, quien ha sido usuaria de dichos servicios, relató: "Ahí en Mitras estaba la escuela en que estudié el Braille. Antes era nada más de discapacidad visual ahora es Centro de Atención Múltiple por los nuevos cambios de la ley de la Educación." 
para poder incorporarlos después a escuelas regulares. Los cursos para bebés y niños los capacitan en el manejo de texturas, la lectura braille, la lateralidad y el espacio y el tiempo.

A continuación, presentaremos un análisis de las instituciones hegemónicas que brindan servicios a invidentes en el AMM. Estas instituciones son consideradas como hegemónicas debido a que son las que mayor reconocimiento e impacto tienen entre las personas ciegas además son las que han perdurado más tiempo y han logrado constituirse como Asociaciones de Beneficencia Pública.

\section{Instituciones enfocadas a la integración laboral en el Área Metropolitana de Monterrey}

En Nuevo León predominan dos modelos de integración laboral para invidentes que han encontrado respaldo de organismos públicos y privados. Tienen raíces sociopolíticas distintas y surgieron en diferentes momentos coyunturales. El modelo más antiguo data de la década de los años cuarenta y se basa en la integración de los invidentes a un trabajo informal; el segundo es de origen más reciente y persigue la integración de los invidentes al sector productivo en general. Para analizar los logros y limitaciones de estos modelos tomaremos en cuenta la perspectiva de tres invidentes cuya integración productiva se ha basado en uno de los dos enfoques.

Los diversos modelos de integración laboral no son meras técnicas de integración social sino se sustentan en discursos socialmente creados acerca de la relación entre la 'normalidad física' y la discapacidad, las capacidades y limitaciones de los invidentes frente a las exigencias del mercado laboral, la posición de los discapacitados en la sociedad y las necesidades y los derechos sociales de los discapacitados. Sin embargo, estos discursos sólo pueden surgir porque las condiciones de vida que encuentra un sujeto en su entorno no le proveen de todos los recursos necesarios para llevar a cabo sus actividades (Hahn cit. en Putnam,
2005:189). Y al revés: las condiciones del entorno parten de un sujeto con determinadas cualidades psicofísicas.

Cuando hay una incongruencia entre entorno y sujeto surgen narrativas que pretenden legitimar la exclusión de un sujeto como 'anormal', 'atípico' o que - desde la perspectiva de los excluidos - buscan revertir su marginación. Ambos relatos acerca de los 'sujetos especiales' se proponen influir la acción de los 'otros'. Se trata, por lo tanto, de políticas que hacen uso de discursos identitarios, es decir, de 'políticas identitarias (Parker, 2005:53ss; Putnam, 2005:188ss).

Los discursos identitarios tienen un efecto doble: por un lado informan al público en general acerca de las características de los 'otros' (por ejemplo, los invidentes) y encaminan a personas e instituciones hacia ciertas formas de apoyo. Por el otro, actúan sobre las construcciones identitarias de los sujetos nombrados (los invidentes) mismos y moldean sus expectativas hacia los demás (por ejemplo, acerca de los apoyos que les deberían de brindar) y hacia si mismos (a lo que pueden aspirar en la vida; lo que son capaces e incapaces de hacer, por ejemplo). Por consiguiente, es posible afirmar que las políticas identitarias representan al mismo tiempo modelos de socialización y de integración social.

Si bien es cierto que los discursos identitarios son, por lo general, autorreferenciales, es decir, articulan una serie de concepciones acerca del sujeto que habla de si mismo (Parker, 2005), también pueden ser utilizados para influir las acciones con relación a otros construyéndolos bajo una cierta luz. Las políticas identitarias se materializan de esta forma no sólo en un discurso sino también en una práctica. Es por esto que hacemos un análisis de los modelos de integración laboral de invidentes intentando recuperar de ahí los preceptos básicos que regían en torno a los invidentes en un momento histórico determinado.

Ante la volatilidad de los discursos (Foucault, 2002), los discursos necesitan ser reproducidos incesantemente por los actores. La reproducción tiene lugar por medio de formas de intervención 
sociopolítica, como por ejemplo, las políticas sociales; al igual que en la acción de los invidentes mismos cuya integración laboral es guiada en uno de estos modelos.

En vista de que los dos modelos que analizamos en seguida surgieron en épocas históricas distintas y en medio de formaciones discursivas disímiles, se ligan a políticas identitarias diferentes que fueron dirigidas en cada época hacia los invidentes y que producen en ellos, por lo mismo, efectos identitarios divergentes.

El análisis de cada modelo busca, por consecuencia, rebasar el mero nivel técnico e incorporar las políticas identitarias contenidas en el mismo así como las correspondientes construcciones identitarias de los ciegos involucrados. La información no se encuentra de forma explícita en la realidad social sino es contenida en los relatos de los invidentes acerca de su historia laboral.

Para construir los relatos, los sujetos hacen uso de un vocabulario, una gramática y estrategias retóricas y narrativas. La deconstrucción de estos elementos permite descubrir los presupuestos y sobreentendidos (Ducrot, 1982) que forman el tejido oculto de las identidades y formas de interacción.

Por último, es preciso enfatizar que a pesar de que los diversos enfoques de integración laboral y los discursos y políticas identitarias surgieron en momentos históricos diferentes, ello no significa que los modelos y discursos más antiguos se hubiesen erradicado. De hecho, los diversos discursos siguen subsistiendo en la actualidad y reclaman validez ya que todos siguen estructurando la vida de ciertos grupos de invidentes.

\section{Las características de la Asociación de atención a invidentes basada en el trabajo informal}

Esta Asociación se inició en los años cincuentas bajo una visión asistencialista y filantrópicosocial. Los invidentes que participan se consideran individuos excluidos, marginados y rechazados por la sociedad y lamentan que las políticas públicas no responden a la problemática de los invidentes.

El modelo de la informalidad laboral relata de la represión y exclusión que grupos sociales y empresas ejercían contra los invidentes en las décadas de los cuarentas hasta mediados de los setentas. Trinidad, por ejemplo, no pudo inscribirse, en aquel entonces, en una escuela secundaria en Monterrey. Para iniciar su educación secundaria tuvo que cambiarse de ciudad. Esta experiencia indica, empero, que los discursos acerca de las limitaciones de los invidentes no eran tan consistentes y homogéneos sino variaban en intensidad entre un lugar y otro. Fueron estas fisuras que permitieron a Trinidad proseguir su educación.

Pero no sólo las escuelas regiomontanas se rehusaron a aceptar niños y jóvenes invidentes, tampoco las empresas mostraron disposición alguna a integrarlos laboralmente. Según Teodoro, ante la falta de oportunidades de estudio y de trabajo, los invidentes sólo tenían como posibilidad pedir dinero en las calles. No obstante, tampoco ahí eran aceptados y pronto la policía los empezó a detener y encarcelar. La exclusión social de los invidentes de todos los espacios públicos regiomontanos se armó así de la violencia física y simbólica con tal de desaparecerlos de la vida pública.

La experiencia vivida por muchos invidentes a mediados del siglo XX impulsó su integración como un "grupo particular" que compartió problemas comunes y necesidades especificas. Pensaron que organizándose en asociaciones lograrían gestionar líneas de atención ante el gobierno. Poco a poco estas estrategias iniciales se institucionalizaron, una precondición para su perduración en el tiempo.

El primer logro de este grupo ${ }^{3}$ consistió en conseguir que el gobierno les concediera en comodato algunas instalaciones para oficinas y un

3 El nombre no se revela para garantizar la seguridad del grupo. 
presupuesto para la manutención del lugar donde solían reunirse. Además, lograron que el gobierno les permitiera pedir limosnas en la calle.

Desde entonces las actividades en la informalidad laboral se han diversificado. Entre las actividades que realizan actualmente se encuentra: invitar profesionistas a que ofrezcan charlas de temas diversos para los socios; además de otra actividad incorporada recientemente; gestión social (búsqueda de donativos ya monetarios, ya en especie: alimentos, medicinas, recibir a personas que ofrecen trabajo como voluntarios, p.e. jóvenes de universidades privadas que realizan su servicio social) con instituciones educativas, de gobierno y privadas; recaudación de fondos a través de rifas, colectas en los cruceros del centro de Monterrey (esta actividad es conocida entre los miembros como boteo) tocadas en camiones y ofrecer comidas a bajo costo a los miembros de la asociación.

El grupo intenta, asimismo, recolectar medicina, despensa y ropa que son distribuidos entre los miembros de la asociación. Asimismo se preocupa por conseguir consultas médicas y descuentos en las operaciones quirúrgicas a las que se tienen que someter los asociados. Actualmente cuenta con un consultorio y un dispensario médico. Estas actividades se han logrado a través de convenios con el Hospital Universitario, que asigna a un médico para que atienda a los asociados.

Para recaudar fondos la asociación organiza rifas. Los boletos son ofrecidos en venta por los invidentes afiliados en los cruceros del centro de la ciudad de Monterrey. La asociación reparte, además, los cruceros y los camiones para el boteo, es decir, para pedir limosnas. De esta manera se garantiza que cada invidente o grupo de invidentes cuente con un espacio económico exclusivo donde no afronta la competencia de otros invidentes.

Si bien la mendicidad constituyó la única salida para sobrevivir la marginación social, al mismo tiempo contribuyó a afianzar la estigmatización de los invidentes como personas desvalidas. Más aún, son los invidentes que piden dinero por las calles y cruceros de Monterrey quienes reproducen y reafirman la permanencia de su 'anormalidad' que les es asignada por el imaginario social. Se trata de una crítica que ha sido articulada por invidentes que no pertenecen a la asociación.

La asociación está consciente de la polémica que existe al interior de la comunidad de ciegos acerca de su política laboral e identitaria. Dado que se trata de una crítica que pone en duda un estilo de vida desarrollado durante muchos años y bajo presiones sociales extremas, es obvio que los afiliados de esta agrupación despliegan una serie de estrategias discursivas para legitimar su quehacer. Esto se observa con gran transparencia en el discurso de Teodoro cuando constata que:

"Entonces la polémica del ciego en México es que no hay leyes, y el gobierno no se preocupa, siempre yo les he dicho a los gobernantes"

Según Teodoro, la vida de los ciegos mexicanos se encuentra marcada por dos circunstancias fundamentales: la ausencia de leyes que los protegerían y la indiferencia del gobierno hacia los invidentes.

Todo discurso retoma sus enunciados de un imaginario social desde donde construye la realidad que enuncia. En el caso de Teodoro, este imaginario se inserta dentro de una visión paternalista y asistencialista de la sociedad con relación a los ciegos. Para hacer valer su reclamo de una atención paternalista que el Estado y la sociedad mexicana deberían brindar a los invidentes, introduce una extensa 'descripción' de los tratos que la sociedad estadounidense brinda a los ciegos.

La poca disposición de empresarios y administradores públicos a integrar a invidentes en sus empresas es la causa principal, según Teodoro, de la expulsión de los ciegos a la calle que ante la discriminación laboral experimentada se convierte en la única fuente de sobrevivencia. Sin embargo, esta solución defendida por los invidentes ha 
sido criticada por el propio Estado como fomento a la mendicidad y la holgazanería.

Frente a las opciones laborales que se les habían ofertado, el trabajo por cuenta propia en la calle o los camiones surge como un espacio de autodeterminación y libertad que el grupo está defendiendo en contra de los múltiples críticos. El 'boteo' expresa, de esta forma, también un acto de resistencia que, como se verá en el siguiente fragmento discursivo, sirve de plataforma para negociar con el gobierno otras opciones laborales.

Si bien el trabajo en la calle es identificado como un espacio laboral con mayor grado de autodeterminación e ingreso les impone un costo moral:

"...mira yo también soy profesionista y me da vergüenza. Porque sí, me da vergüenza, porque a veces ando "coopera, coopera". $Y$ me encuentro ahí en la calle gente que me dice: "¿Cómo está licenciado?" Y yo: "pérate, 'pérate, me pones en ridículo, hombre". Y hasta licenciado me dicen.."

\section{Trinidad}

El fragmento anterior tiene además, una clara función pragmática que se liga al hecho de conversar con una profesionista (por lo tanto, un miembro del mismo gremio profesional) y dando por supuesto que los profesionistas no trabajan en las calles, sino en oficinas. La vergüenza admitida adelanta una posible condenación moral de parte de la interlocutora cancelándola de esta manera. En la situación de la entrevista el relator reivindica así su cualidad profesional. El tema de la vergüenza cubre así varias funciones pragmáticas: constituye una denuncia del costo emocional y social que la exclusión social infringe a los invidentes profesionistas; permite presentarse frente a la interlocutora como persona culta y significa así una autoreivindicación social del relator frente a la entrevistadora.

Finalmente, la expulsión de los profesionistas ciegos al trabajo en la calle refuerza el argumento central de este grupo de invidentes de que no encuentran ante si un campo de desarrollo laboral y que siguen constituyendo un sector de la población cuyas necesidades son desatendidas por el Estado.

\section{Construcciones identitarias y trabajo en el sector formal}

Desde mediados de los años ochenta, se creó un tipo de asociación civil que se ocupa de la ceguera y los problemas de los discapacitados. Este modelo nació a partir de inquietudes científicas: se proponían hacer investigación biomédica y ofrecer asesoría médica a familiares y afectados por la ceguera. Sin embargo ante la falta de recursos para financiar la investigación, resultó difícil sostener estos objetivos. En adelante nos vamos a concentrar en una asociación de invidentes en particular, cuyo nombre no será revelado para proteger la identidad de sus miembros.

Durante la década de los noventa y gracias a la inserción de esta asociación en redes mundiales de apoyo a discapacitados, se familiarizó con modelos de atención que se habían implementado con éxito en países como Suiza, España o Estados Unidos. El contacto con agrupaciones internacionales le abrió, además, la puerta a nuevos conocimientos científicos y avances tecnológicos. Logró establecer, incluso, programas de intercambio con asociaciones estadounidenses y empezó a enviar a invidentes a Estados Unidos donde estudiarían una carrera.

En el caso de esta asociación se observaron cambios de membresía significativos. Durante los ochenta se observó una intensa participación de médicos; en cambio, desde la década de los noventa se afiliaron sobre todo padres de familia que tienen hijos invidentes y adultos invidentes. Ellos se encargan del funcionamiento de la asociación y de las tareas organizacionales. Un rasgo distintivo de esta asociación constituye el nivel socioeconómico de los integrantes de su consejo directivo que se integra en su mayoría por personas de clase media alta y alta que cuentan con estudios profesionales en escuelas privadas. 
La extracción social de los miembros fundadores facilitó a la asociación establecer contactos con empresarios y políticosal igual que con asociaciones de invidentes en otros países y con escuelas para invidentes en el extranjero. La transferencia de conocimientos y tecnologías que se posibilitó a través de estos canales y lazos formales e informales influyó profundamente las formas operativas y los programas de atención adoptadas.

Las actividades de la organización se encuentran marcadas por el discurso de la integración social de los invidentes que abarca tanto la inclusión al sistema educativo, al sector laboral como a otros espacios sociales. Se propone como misión erradicar todas aquellas actividades laborales y sociales que explota la condición de invidentes para conseguir fondos. La asociación espera poder impulsar así un cambio en las actitudes de los mismos invidentes para que dejen de vivir de la caridad pidiendo limosnas y logren insertarse en un trabajo productivo. Este tránsito es identificado como un paso hacia la emancipación de este grupo social. La asociación resumió este objetivo de la siguiente forma:

"Se busca concientizarlos (a los invidentes asociados, B.B.) de que son capaces de desempeñar trabajos dignos que les provean una remuneración económica, buscando que no se vuelvan personas que dependan económicamente de los demás" (ITESM, 2001:215)

Un trabajo es considerado digno cuando genera a los invidentes una remuneración, es decir, un sueldo o un salario. A través de esta categoría se sobreentiende que la asociación busca la inserción de los invidentes al sector formal de la economía. La creación de una base económica constituye la precondición para lograr la independencia no sólo económica sino también social de los invidentes. Para lograr su incorporación laboral y social los invidentes deben de desarrollar sus capacidades individuales, pero sobre todo la autodisciplina ${ }^{5}$. Para que no sólo niños sino también los adultos se formen en la autodisciplina se les exige constantemente llegar temprano al trabajo y cumplir con la producción establecida, y mejor aún, sobrepasar las metas productivas.

Lo llamativo del modelo de atención establecido por esta asociación de invidentes consiste en la integración del discurso en torno al Estado de Bienestar con el ideario del neoliberalismo. Esto se observa con claridad al impulsar la asociación por un lado la aceptación de las personas discapacitadas en el sector laboral. Por el otro, empero, ratifica que este logro inicial sólo se consolida si los invidentes desarrollan una autodisciplina laboral que garantice su permanencia en el trabajo y cuando continúan perfeccionando sus capacidades individuales y su sentido de responsabilidad. Como un estímulo al desarrollo de la responsabilidad la asociación ha establecido una cuota para los socios que debe ser cubierto religiosamente.

\section{Actividades desarrolladas por la Asociación}

Las actividades desarrolladas por la asociación cubren una amplia gama de servicios. Se ocupa de tareas educativas, creación de oportunidades laborales y ofrece, además, una amplia variedad de servicios. En el campo educativo la asociación ofrece tanto estudios básicos como algunos cursos de orientación, movilidad y entrenamiento en el sistema braille. Adicionalmente facilita el acceso a tecnología (capacitación en computación con programas especiales para personas invidentes) avanzada.
4 Debemos resaltar que ello no implica que todos los integrantes de las Asociaciones en este modelo sean de clase alta sino que, según se observo es más bien el Consejo Directivo mientras que los socios en su mayoría pertenecen a la clase media, media baja y baja.
5 Esta tesis del individuo autodisciplinado es desarrollada por Gilles Deleuze en el artículo "Las sociedades de control". 
Entre los servicios se encuentran, además, la orientación y el apoyo psicológicos al igual que servicios de fono y biblioteca. Un campo importante constituye, también, la distribución de información médica actualizada relacionada con las discapacidades visuales así como la asesoría y la consulta médicas especializadas.

La integración laboral es organizada a través de un programa de capacitación que gira entorno a un "taller protegido" donde los socios reciben entrenamiento y capacitación laboral. Una vez alcanzado el nivel de preparación laboral, la asociación contacta a empresas para que los invidentes logren insertarse en una actividad productiva formal. Esto sucede, empero, solamente si los invidentes así lo desean.

Los invidentes entrenados a través del taller tienen el compromiso de aportar a la asociación la décima parte de su salario que reciben por su trabajo en el taller. Este diezmo persigue, por su parte, una función educativa. Se trata de que los invidentes:

"... aprendan hábitos de responsabilidad, hábitos de cumplimiento, hábitos de cooperación mutua. No fomentamos nada que tenga que ver con la flojera ni con la dependencia" (ITESM, 2001: 222)

En el momento en que un invidente cuenta con una oferta laboral de una empresa, la asociación evalúa si necesitará contar con equipo especializado para lograr su mejor adaptación posible al área de trabajo y consigue el equipo y lo instala en la empresa contratante en caso de ser necesario.

\section{El concepto cooperativista como alternativo de integración económica y social: el caso de SOCOININ}

Hasta ahora hemos revisado los conceptos de discapacidad y los procesos identitarios con relación a dos tipos de organización material de la vida de invidentes en el Área Metropolitana de Monterrey. El primer grupo vive de dadivas que pide en los cruceros de las grandes avenidas o canta en camiones y la vía pública con la esperanza de recibir alguna retribución de los transeúntes. El segundo grupo de invidentes labora en una maquiladora que fue creada por una asociación civil con el objetivo expreso de ofrecerles una alternativa de empleo a invidentes y brindarles un sustento material orientado en la asalarización. En las siguientes líneas abordaremos una tercera opción de integración laboral: talleres artesanales. En concreto, analizaremos el caso de la Sociedad Cooperativa de Invidentes Independientes (SOCOININ).

Esta cooperativa se constituyó debido a la insatisfacción experimentada por sus integrantes con el trabajo informal y con diversas formas de integración socioeconómica. Los cooperativistas consideran que los programas y modelos de apoyo a invidentes en el Área Metropolitana de Monterrey no promueven ni fomentan en la realidad la independencia económica de los invidentes ni tampoco respetan los derechos fundamentales y humanos de los invidentes.

El rechazo hacia las relaciones sociales verticales y la convicción de que los procesos democráticos, la igualdad y la solidaridad deben de regir las relaciones y las acciones entre los diversos actores se cristaliza en la organización de la asociación misma que por estas consideraciones éticas no cuenta con una mesa directiva. Todos y cada uno de los integrantes participan en la toma de decisiones. Las funciones de cada miembro en la cooperativa se establecen a partir de las aptitudes que cada uno presenta. Por ejemplo, Ambrosio es considerado como una persona creativa ("es muy ingenio", señalan) por lo que él se encarga de la capacitación y la introducción de nuevos productos. En cambio, Anselmo es percibido como una persona desenvuelta, una característica que le predestina para las relaciones públicas y la consecución de nuevos clientes. 


\section{Características de la Cooperativa}

La cooperativa cuenta con cinco miembros quienes se dedican a la fabricación de productos de limpieza: escobas, trapeadores y aviones para limpiar ventanas, entre otros. A pesar de trabajar juntos desde hace un par de años, la sociedad cooperativa se constituyó en el plano legal apenas en el año 2003.

Al constatar en el primer enunciado que el grupo de invidentes ha sido innovador en cuanto a la creación de un sustento material no conocido hasta entonces ("Nosotros somos los pioneros"), el relator fundamenta en lo siguiente su aseveración presentando este colectivo de invidentes como un grupo dispuesto a retar los estereotipos sociales y el imaginario social predominante acerca de la discapacidad visual. En calidad de 'innovadores' el grupo empezó a transformar prácticas acostumbradas y abrió nuevos caminos. El grupo retó al discurso dominante en torno a los invidentes de dos maneras: (a) a través de su inserción en el proceso de producción fabril y (b) a través del uso de maquinaria y herramienta considerada de peligrosidad para invidentes.

Cuando un individuo o un grupo irrespetan convenciones establecidas o ponen en duda una serie de presupuestos comúnmente aceptados enfrentan, por lo común, cierta resistencia en el entorno. $Y$ efectivamente, el discurso hace referencia a un grupo opositor referido en el relato a través del pronombre personal "ellos". Si bien Armando no describe en términos sociodemográficos a estos críticos (funcionarios, periodistas, empresarios, etcétera) los caracteriza a través de su imaginario acerca de personas con discapacidad. Eran personas que daban por supuesto que la discapacidad visual era incompatible con el uso de cierto tipo de instrumentos de trabajo, en concreto, con tijeras y maquinas eléctricas ("...icómo iba a ser posible de que los ciegos trabajamos en esa forma").

El discurso de los miembros de SOCOININ manifiesta una concepción acerca de la atención social a los discapacitados que es muy distinta de las expectativas de los invidentes que viven del trabajo informal (como limosneros y cantantes). Se trata de un distanciamiento no solamente social sino también político que se observa en el campo deíctico: al referirse el narrador a "nosotros", incluye solamente los miembros de SOCOININ y excluye a otros grupos de invidentes con concepciones distintas de cómo organizar su sustento económico.

A diferencia de sus colegas que trabajan como asalariados en una maquiladora, el colectivo de SOCOININ se identifica intensamente con su empresa.

\section{"Se siente orgullo, siempre decimos mi em- presa, mi cooperativa"}

La importancia que adquiere la cooperativa en el bienestar mental y social de este grupo de invidentes se encuentra íntimamente relacionada con experiencias ligadas a la filantropía empresarial en fábricas donde habían laborado en el pasado. La única excepción constituyó la empresa "La Americana":

"Armando: Anselmo trabajó en una empresa, pero, pues, con muy malos recuerdos, gente que trata de explotar porque al cabo eres ciego y te voy a dar una limosna. Tuvimos esa experiencia amarga. Haz de cuenta que nos estaban dando migajas, de que nos hacían un gran favor en tenernos empleados... Andrea: ...pero si los estaban explotando. Entonces ellos dicen: 'Lo mío no lo dejo porque esto es mío y aquí trabajo lo mío.' Entonces tienen razón.

El discurso de Anselmo no constituye sólo una crítica a la filantropía sino una denuncia del altruismo como una estrategia empresarial para explotar a los invidentes: "... gente que trata de explotar porque al cabo eres ciego y te voy a dar una limosna... Haz de cuenta que nos estaban dando migajas, de que nos hacían un gran favor en tenernos empleados...". A diferencia de otras 
formas de explotación económica, en el caso de los ciegos se espera incluso que el mal pago (... nos estaban dando migajas...) sea agradecido por los trabajadores invidentes dado que se les hizo "un gran favor en tenernos empleados". La explotación se disfraza de caridad social que no puede ser reclamada por los afectados ya que se les es presentada como un acto de bondad empresarial.

SOCOININ se fundó justamente como un proyecto alternativo a esta práctica empresarial. Pretende ofrecer un trabajo digno que respete los derechos laborales y sociales y que genere una remuneración justa. El proyecto de cooperativa introduce así una diferenciación entre la justicia social y las acciones caritativas o asistenciales.

Pero el trabajo en la cooperativa ofrece, además, otro beneficio: permite la autonomía y la autodeterminación de sus integrantes ("....no hay quien diga: 'Oye, iporqué estás sentado? Oye ¿por qué no hiciste?'). Si bien los cooperativistas pueden no presentarse un día en el trabajo por las razones que tengan, al momento de afrontar una demanda urgente el colectivo puede contar con cada uno ("... pero si llega el apuro de que hay un pedido fuerte, trabajamos...")

"Somos nuestros propios patrones. Si dices: "hora no tengo ganas, 'hora no trabajo', pero si llega el apuro de que hay un pedido fuerte, trabajamos... Entonces esa es la diferencia de trabajar con lo de uno, pos, se agarra con más cariño y, como te digo, pues, no hay quien diga: 'Oye, ¿ porqué estás sentado? Oye ¿por qué no hiciste?' Porque sabemos a que horas trabajar..."

A diferencia del trabajo fabril donde se les exige a los asalariados una producción intensa y extenuante que, en caso de interrumpirse para tomar un descanso, produce el reclamo de un supervisor, la cooperativa es experimentada como un espacio de libertad ("...esa es la diferencia de trabajar con lo de uno, pos,...").

A diferencia del concepto empresarial, en tanto, que identifica a la producción fabril exclusiva- mente como un espacio productivo, los cooperativistas no reducen su trabajo a aspectos materiales sino enfatizan asimismo la integración social y la solidaridad del grupo. El trabajo es un espacio de convivencia y de esparcimiento que se celebra, por ejemplo, cada viernes mediante una comida a costo del taller:

"Nosotros pusimos el viernes hacer el sacrifico de que la fabriquita nos diera de comer, comprar comida para no traer nuestro lonche"

El principal problema que enfrenta la cooperativa es el de la comercialización de los productos. Si bien SOCOININ cuenta con algunos clientes fijos - situación que permite la subsistencia del negocio - las expectativas del grupo rebasan la actual capacidad laboral de la cooperativa que por el momento no les permite emplear a más personas discapacitadas. El estancamiento del negocio es atribuido por los miembros de SOCOININ a la escasa solidaridad de la sociedad y, en particular, de las instituciones públicas y privadas con los discapacitados.

\section{CONCLUSiOnes}

Las personas ciegas, ante la carencia de oportunidades que promuevan su inserción social, despliegan estrategias de afrontamiento (mediante la formación de Asociaciones) orientadas a la creación de recursos útiles para la reinserción a las actividades cotidianas y a las productivas. La creación de redes de apoyo, la lucha por obtener una formación técnica o profesional, la insistencia colectiva en la obligación del Estado de generar una infraestructura que garantice la igualdad social que la Constitución otorga a todo ciudadano, son ejemplos de estrategias encaminadas a nivelar las condiciones de existencia de las personas ciegas con la de las normales y a mitigar la exclusión y estigmatización de parte del contexto social. 
Aun cuando en el Área Metropolitana de Monterrey se han incrementado el número instituciones que ofrecen cursos (orientación y movilidad; actividades manuales, entre otros) para personas ciegas, siguen siendo limitados en cuanto a movilidad socioeconómica. Faltan oportunidades para el desarrollo técnico y profesional, como por ejemplo la masoterapia, actividad que no sólo ofrece posibilidades económicas y laborales, sino que es básica e indispensable para el desarrollo del reconocimiento de las capacidades y habilidades (p.e uso del tacto) de las personas ciegas.

Algunas personas ciegas reproducen el imaginario social en el que se considera que la vista es el sentido imprescindible para desarrollarse como un miembro 'competente' de la comunidad. Esto se complejiza debido a que en las sociedades occidentales el uso del tacto en la exploración del propio cuerpo y -más aún- del de otros, se encuentra en extremo restringido. Una persona que toca a otra persona sin consentimiento expreso puede incluso ser sancionada legalmente, al invadir y violar el espacio personal de un determinado individuo: su individualidad y privacidad. Una sanción similar no existe para la mirada, a pesar de que pueden resultar igualmente invasivas, intrusivas e incluso agresivas y violentas. Esta experiencia se articula en dichos populares como "hay miradas que matan", o bien, "me sentí violada por su mirada".

Los datos empíricos recabados, sugieren que las personas con discapacidad enfrentan una es- pecie de negación de reconocimiento con relación a sus cuerpos. Ello inhibe y reprime el desarrollo de sus capacidades y habilidades para conocer y aprehender el mundo. De ello se deriva una dependencia hacia las personas que cuentan con el sentido de la vista y que resulta difícil de superar. A pesar de la importancia de este tema, la exploración de estas experiencias ha sido relegada en la investigación académica, al igual que en la discusión de las propuestas de intervención.

\section{BiBLIOGRAFÍA}

Basaure, Mauro (2011) Reificación y crítica de las patologías sociales en el marco del proyecto teoría critica de Axel Honneth. Enrahonar, 46: 75-91.

Bustos, Brenda, Sieglin, Verónica (2006) Discapacidad, formas de inserción laboral y construcciones identitarias en el Área Metropolitana de Nuevo León. Pautas hacia el replanteamiento de políticas sociales dirigidas a invidentes. Tesis de Maestría Facultad de Trabajo Social, UANL.

Honneth, Axel (2007) Reificación. Un estudio en la teoría del Reconocimiento. Katz Editores, Buenos Aires.

Honneth, Axel (2009) Patologías de la razón. Historia y actualidad de la teoría critica. Katz Editores, Buenos Aires.

Honneth, Axel (2009) Crítica del Poder. Fases en la reflexión de una Teoría Critica de la sociedad. Ed. A. Machado Libros, Madrid, España.

Honneth, Axel (2010) Reconocimiento y menosprecio. Sobre la fundamentación normativa de una teoría social. Katz editores, Buenos Aires.

Renkema,Jan (1999) Introducción a los estudios sobre el discurso. Editorial Gedisa, Barcelona.

Ricoeur, Paul (2006) Caminos del reconocimiento. Tres estudios. Fondo de Cultura Económica, México. 